\title{
The Socio-cultural Dynamics of Antibiotic Misuse in Hyderabad City, India: A Qualitative Study of Dentist and Pharmacist
}

\author{
Shoeb Ahmed ${ }^{1}$ \\ Centre for Economic and Social Studies, Begumpet, Hyderabad-500016.
}

\section{Correspondence:}

Contact: shoebilyas@gmail.com, +91-7032771303

\section{ABSTRACT}

Introduction: Antibiotic resistance and antibiotic-associated adverse events are rapidly escalating and considered as a global health threat and public health problem. There is poor evidence base on the contextual specificities and everyday use of antibiotics in public health dentistry and pharmacy dispensing practices. The study explores the socio-cultural dynamics emergent around antimicrobials in the Indian Public dentistry and pharmacy practice in India.

Methods: Using purposive sampling, dentists and pharmacists were recruited for the study in Hyderabad City, Telangana State, India. Using semi-structured interviews, dentists and pharmacists were asked about how antibiotics were prescribed and dispensed, perceptions of antibiotic use in community and socio-cultural norms prevailing in the use of the antimicrobial practice.

Results: The dominant themes, emerged by both dentists and pharmacists, was the avoidance of dentist visit on encountering dental infection, reliance on self-medication, use of over-the-counter antibiotics and easy accessibility of antibiotics without prescription as a primary driver of antibiotic misuse and development of antimicrobial resistance, which is associated with socio-cultural practices and economic problems.

Conclusion: The speculations of qualitative responses to antimicrobial resistance are profoundly entangled in the specificities of dental infection management across cultures and locales in daily life. This study emphasizes the need for enforcement of regulatory mechanisms on antibiotic dispensing over the counters, educating dental patients in avoiding self-medication, educational and training initiatives are necessary to sensitize and rationalize dentist and pharmacist in the use of antibiotics.

Keywords: India, Antibiotics, Antimicrobial Resistance, Qualitative interviews, Dentist, Pharmacist.

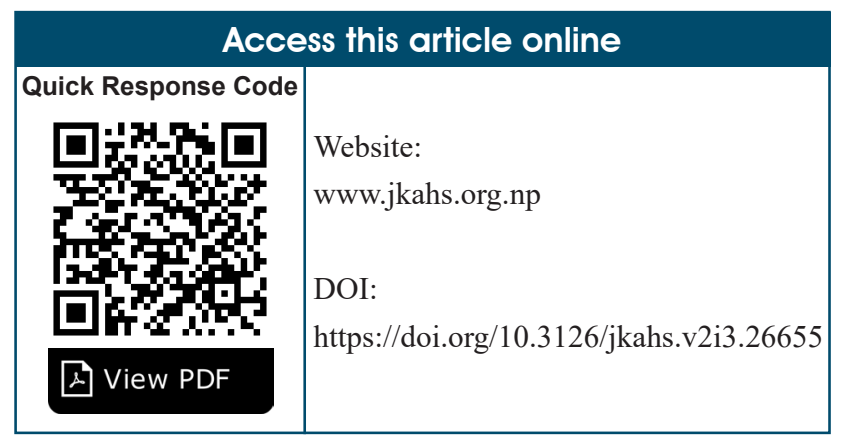

\section{Article Info.}

\section{How to cite this article?}

Ahmed S. The Socio-cultural Dynamics of Antibiotic Misuse in Hyderabad City, India: A Qualitative Study of Dentist and Pharmacist. Journal of Karnali Academy of Health Sciences. 2019;2(3): 197-202.

Received: 11 Oct., Accepted: 23 Nov., Published: 11 Dec. 2019

Conflict of Interest: None, Source of Support: None

\section{INTRODUCTION}

Antimicrobial resistance (AMR) is emerging and increasing global public health threat challenge due to misuse and overuse of antibiotics, limited immediate prospects of research and development of new antibiotic classes $^{1-5}$. Outcomes of ecological studies have shown that the resistance at the population level 
is due to overuse of antibiotics. ${ }^{6-7}$ Dental professionals prescribe antimicrobial agents for conditions both therapeutically and prophylactically ${ }^{8}$. Dentists prescribe more or less $10 \%$ of outpatient antibiotics 9 Studies suggest that $30 \%$ to $50 \%$ of prescribed antibiotics are needless or not optimally prescribed. ${ }^{10}$ Dentist prescribes antimicrobials for about all conditions the patient present's with ${ }^{11}$ and there are no national and international guidelines for the treatment of specific dental infections in dentistry, but there are interventions that can be done as first-line treatments instead of prescription of antibiotics. ${ }^{12}$

\section{Significance of Antimicrobial Resistance in India}

AMR is declared as global health threat for the twenty-first century ${ }^{3}$ and estimates by 2050 , indicate cost of USD $\$ 100$ trillion burden on global economy, as infections from resistant bacteria will surpass cancer as the leading cause of death and in India, the burden of antimicrobial resistance will be one million lives per year ${ }^{13}$ due to failure of implementation of comprehensive surveillance of antibiotic usage ${ }^{14}$ and resistance to accepting the empirical realities of 'antimicrobial misuse' which are rooted in cultural practices and structural environments in India. Previous studies in India on public knowledge and awareness on antimicrobial resistance is inadequate and unequally distributed. ${ }^{15-16}$ This study aimed to evaluate the socio-cultural dynamics emergent around antimicrobials use in the Indian Public health dentistry and pharmacy practice in Hyderabad, India.

\section{METHODS AND FIELDWORK}

This study is a qualitative study done using purposive sampling, privately practicing dentists and pharmacists with varying socioeconomic profiles in urban and rural neighborhoods were recruited for the study in Hyderabad city, Telangana State, India. Participants were approached at their respective places of work where they were given information about the study by the interviewer and had an opportunity to ask questions. Ethical clearance was obtained from Centre for Economic and Social Studies, Hyderabad,
Telangana, India. Informed consent was taken before the interview. The study involved 25 participants, including 19 dentists ( 7 female and 12 male) and 6 pharmacists ( 2 female and 3 male). Participants' ages ranged from 23 to 60 years, with the majority of dentist in the age group of the $30 \mathrm{~s}$ or $40 \mathrm{~s}$ and the majority of pharmacists in their 20 s or 30 s.

The semi-structured interviews which lasted for 45 to 60 minutes were digitally audio-recorded and were fully transcribed. Interviews began with general questions on participants' experiences of oraldental infection management, how antibiotics were prescribed and dispensed, perceptions of antibiotic use in community and socio-cultural norms prevailing in antibiotic use, their daily basis sensitivity towards antibiotic resistance and the self-reported influences on the use of antibiotics in their day to day practice. All interviews were done in the English language.

\section{Context of the Study}

Hyderabad is the capital city of southern India's Telangana state with 217 areas Sq.km, 16 mandals; one town surrounded by 67 villages and has a population of 6.8 million 17. Hyderabad has significant socioeconomic, linguistic, and religious diversity. In Hyderabad, the reported urban poverty has been measured at $23 \%$ of the total population ${ }^{18}$. The health care system in Hyderabad is a mix of both private and public health care facilities with standardized and easily affordable health care than many cities in India ${ }^{19}$. Hyderabad citizens prefer treatment at the private health care sector and approximately $28 \%$ of citizens use public health care facilities, due to poor quality of patient care, extreme waiting time and far distance locations ${ }^{20}$.

\section{Analysis}

The methodology for this study was derived from the constructivist grounded theory within qualitative research $^{21}$. The basic aim was to attain a comprehensive understanding of the perspectives presented in interviews and to place them within a spectrum of broader core beliefs, agendas, and participant's life 
experiences. The interviews were analyzed based on initial thematic analysis. After identification of theme, the interviews were further analyzed for other related comments to develop themes further and finally reviewed the literature to identify conceptual tools to make sense of the patterns derived from data.

\section{RESULTS}

\section{Self-Medication/Use of Over-the-Counter Antibiotics based on the Recommendation by a Pharmacist.}

The interviews focused on participants' basic understandings of antimicrobial practices, and their perceptions of the reasons behind an antibiotic misuse and resistance. The dominant theme, emerged by both dentists and pharmacists, was the avoidance of dentist visit on encountering dental infection and reliance on 'self-medication'/ use of over-the-counter antibiotics based on recommendation by pharmacist and easy accessibility of antibiotics without prescription as a primary driver of antibiotic misuse and development of antimicrobial resistance which is associated with social, economic and cultural practices and problems.

\section{Interviewer: Why Antibiotic Resistance is Arising?}

Participant: it is mainly because of intolerable dental pain.... non-affordability of dentist consultation fees and time constraints to visit dental clinic, they ask for medications within their budget and we dispense the antibiotics within their budget and recommends them to use for at least three days, but most patients buy for one day or two days and latter they will not turn up to buy further and they keep the medicine cover and use it repeatedly.... If they don't get the relief they visit the dentist. [Private Pharmacist, 36, male]

The above reflections emphasize on the underlying structural drivers like immediate relief from dental pain, accessibility of antibiotics over the counter without dentist prescription, affordability of dentist consultation fees, and time constraints to visit the dental clinic are the primary reasons for the misuse of antibiotics.

The availability of hand in cash is a big issue. As many private pharmacies maintain kaatha's (book of accounts of customers)...... It makes easy for people to visit pharmacy without cash in hand and ask for antibiotics for dental pain / for any other illness problem....... Customers also return antibiotics in case dental pain subside.... Ask to replace antibiotics.... Even without taking for prescribed schedule...... Mutual trust and arrangements ...... easy monthly EMI options...will retain the pharmacy customers. [Pharmacist, 44, Male, Pharmacy owner]

. . . the other common factors [for overuse and development of antibiotics resistance] customers/ patients lack time to commute and consult the dentist, as most dental clinics open by 10.30 am to 11 am and having office reporting time constraints, waiting time at dental clinic, high dental consultation fees in private dental clinics, all the above factors lead to making patients buy antibiotics over the counter and use the same antibiotics repeatedly as and when they feel dental pain. [Pharmacist, 29, Female, Pharmacy Supervisor]

The above situations described by study participants highlight various dimensions of AMR like unprescribed dispensing of antibiotics over the counter, taking an incomplete course of treatment and repeated intake as and when needed sometimes leading to overconsumption are all responses to structural constraints and non-compliance to standard clinical norms.

In oral health care service delivery there exist commercial component, even though people may not perceive it as commercial activity. Unfortunately, the dentist faces pressure and caught between counteracting forces between patients for quick results and pharmacy owners to prescribe generic drugs and a lot of supplements in the form of multivitamins, mouthwashes......[Pharmacist, 52, Pharmacy owner] 
The nexus that study participants expressed was the occurrence of commercial interests, and the pressures experienced by the dentist at the command of pharmacy owners. As most pharmacy retailers support dentist in buying types of dental equipment, paying their dental clinic rentals, electric bills, and routine consumables' like injections, gloves, cotton, anesthetic cartridges, and helpers to assist them and even some dental practices are accommodated within the pharmacy premises for free of cost, in all the above scenarios dentist prescriptions contribute to the revenue of pharmacy retailers turnover and they depend on dentist prescriptions which have the value on average of above 300-600 rupees and the common contents of prescription are antibiotics, analgesics, antacids, multivitamins, toothpaste, mouth wash, and other supplements like inter-dental brushes. The dentist has to maintain the high prescription value irrespective of the actual need to maintain good nexus with a pharmacist who is often also the referrers of dental patients. The above mentioned 'counteracting forces' include the structural conditions around antibiotic misuse and the nexus between dentist and pharmacy retailers.

\section{Socio-Cultural Dynamics of Antibiotics Misuse: 'Counteracting Forces' and the Responses of Dentist and Pharmacists}

The factors contributing to the problems of antibiotic 'misuse' is the result of the interaction of broader social and cultural dynamics. The dentist study participants were aware of the consequences of prescribing antibiotics prophylaxis before dental treatments as the dental patient's willingness to 'dentist shop' viewed as a major constraint on dentist' capacity to promote oral antibiotic prophylaxis before carrying dental treatments.

...If a dentist promotes dental prophylactic antibiotic and asks the patient to take antibiotics course and wait for the completion of the antibiotic regime ... the very next day the patient will go and see a different dentist, patients are not ready to wait for a dental prophylactic antibiotic so they are forced to do dental treatments and prescribe antibiotics after treatment and many patients are not compliant in following dentist prescription post dental treatment which is one of the causes of AMR. [Dentist, 44, Male, Oral Maxillo Facial surgeon, Private Dental Practice]

. . [Dentist] directly prescribes beta-lactams example Tab Augmentin $625 \mathrm{mg}$ in combination with nitroimidazoles example Tab Metronidazole for major dental infections like necrotic pulp with acute apical periodontitis presenting with swelling and with moderate/severe preoperative symptoms. As they want the patient to get a better prognosis and maintain their reputation in a competitive dental market. [Dentist, 28, Male, General Dental Practioner, private dental practice]

The above arguments suggest that dentist face psychological and market pressure to prescribe combination antibiotics for oral-dental infections and need to satisfy and exceed patient expectations. To sustain dental practice, the dentist has to focus on retaining the dental patients.

When the dental patient present to my clinic.... with periapical or periodontal abscess, I presume patient in pain wants to get rid of it as early as possible and expects good outcome with my consultation... I have to prescribe him high-end antibiotic... because of poor oral hygiene conditions and gutka chewing habits ... but if the patient doesn't comply with my prescription regime he can be at great risk of AMR in the future. [Dentist, 36, Male, Periodontist, Private Practice]

Dental surgeons deal with dental infections caused by virulent bacteria. A majority of these infections can be managed by the most common antibiotics... But problems of AMR may arise from dental prescriptions that are given for inappropriate antibiotic, in appropriate circumstances at inadequate daily dosage, and with no initial loading dose. [Dentist, 27, Female, General Dental Practioner, Private Practice]

Antibiotic resistance is a true challenge to stop emerging "superbugs." Fighting with antibiotic resistance is not a one-time affair.... It needs intrinsic motivation and requires sustained commitment and all the dental fraternity must evaluate their antibiotic 
prescribing behaviors for better effectiveness and outcome. [Dentist, 43, Male, Periodontist, Private Practice]

Dentist faces diverse challenges while prescribing antibiotics and dental treatments such as social structural constraints, asymmetry of information between dentist and patient, maintaining professional identity, patient expectations, and affordability of patients.

\section{DISCUSSION}

Addressing antimicrobial resistance in public health dentistry and pharmacy practice call for an examination of how the specificities of antibiotic use are entangled in the specificities of daily life across cultures and locales. This study has explored a few emergent social dynamics of antibiotic use in Hyderabad city in the context of an emerging global threat of antimicrobial resistance. This study discovered that how pharmacist and dentist navigate constraint of market pressures in antibiotics prescription and dispensing which are deeply embedded in survival practices of dental clinics and pharmacies in highly competitive health care markets. Pharmacists dispensed antibiotics improperly in different contexts. Other studies did previously by Kotwani et al. (2011) ${ }^{22}$ showed similar results like a pharmacist inappropriate antibiotic dispensing and use owing to commercial interests and lack of knowledge about the rational use of antibiotics. Kamat \& Nichter $(1998)^{23}$ reported self-medication with an antibiotic.

There are studies which prove that an appraisal of the antimicrobial prescribing habits of dental professionals along with an educational component about antimicrobial resistance have a positive effect on the frequency and use of antimicrobial agents ${ }^{24-25}$ and also raising understanding of antimicrobial resistance amongst urban-rural population and dental /pharmacist fraternity by overcoming the challenges of socioeconomic constraints, cultural norms and practices, different forms of nexus between dentist and pharmacist in oral health care service delivery.

\section{CONCLUSION}

As dentist and pharmacist play important a role in public awareness and understanding of oraldental infections and use of antimicrobials. The dental and Pharmacy fraternity should focus on preserving the currently in use antibiotics through active surveillance, pharmaceutical regulation, and antimicrobial stewardship. This study emphasizes the need for more educational training initiatives to sensitize and rationalize dentist and pharmacist in the use of antibiotics. Future research should take account of the dental patient's experiences and their perceptions of antibiotic misuse and the development of antimicrobial resistance.

\section{REFERENCES}

1. Centres for Disease Control and Prevention [CDCP]. Antibiotic resistance threats in the United States, 2013.

2. Spellberg B, Powers JH, Brass EP, Miller LG, Edwards JE. Trends in antimicrobial drug development: implications for the future. Clin Infect Dis. 2004; 38(9):1279-1286.

3. Reddy S, Jacob J, Varkey J, \& Gaynes R. Antibiotic use in US hospitals: Quantification, quality measures and stewardship. Expert Review of Anti-Infective Therapy.2015; 13(7): 843-854.

4. World Health Organization (WHO). Global action plan on antimicrobial resistance. Geneva: WHO, 2015.

5. Center for Disease Dynamics, Economics \& Policy (CDDEP). State of the World's Antibiotics, Washington, DC, 2015.

6. Goossens H, Ferech M, Vander Stichele R, Elseviers M. ESAC Project Group. Outpatient antibiotic use in Europe and association with resistance: a cross-national database study. Lancet. 2005; 365(9459):579-587.

7. Adriaenssens $\mathrm{N}$, Coenen $\mathrm{S}$, Versporten $\mathrm{A}$, Muller A, Minalu G, Faes C, et al. European Surveillance of Antimicrobial Consumption (ESAC): outpatient antibiotic use in Europe 
(1997-2009). J Antimicrob Chemother. 2011; 66 (suppl 6):vi3-vi12.

8. Tong DC, Rothwell BR. Antibiotic prophylaxis in dentistry: A review and practice recommendations. J Am Dent Assoc. 2000; 131(6):366-74.

9. Hicks LA, Bartoces MG, Roberts RM, Suda KJ, Hunkler RJ, Taylor TH Jr, et al. US outpatient antibiotic prescribing variation according to geography, patient population, and provider specialty in 2011. Clin Infect Dis. 2015; 60(9):1308-1316.

10. Demirjian A, Sanchez GV, Finkelstein JA, Ling SM, Srinivasan A, Pollack LA, et al. CDC Grand Rounds: getting smart about antibiotics. MMWR Morb Mortal Wkly Rep. 2015; 64(32):871-873.

11. Palmer NO, Martin MV, Pealing R, Ireland RS. An analysis of antimicrobial prescriptions from general dental practitioners in England. J Antimicrob Chemother. 2000; 46: 1033-6.

12. Trevor M Johnson, Joanna Hawkes. Awareness of Antibiotic Prescribing and Resistance in Primary Dental Care Prim Dent J. 2014; 3(4):44-47.

13. UK Review on Antimicrobial Resistance (UK RoAR). Tackling drug-resistance infections globally. London: Welcome Trust and the Department of Health (UK). 2016.

14. Sánchez M, Sivaraman S. News Media Reporting of Antimicrobial Resistance in Latin America and India. In Sosa A Byarugaba, D Amábile-Cuevas, C Hsueh, P Kariuki S. and I. Okeke (Eds.). Antimicrobial resistance in developing countries (pp. 525-537). New York, NY: Springer New York. 2010.

15. Chandy S J, Mathai, E, Thomas K, Faruqui A R, Holloway K, Lundborg C S. Antibiotic use and resistance: Perceptions and ethical challenges among doctors, pharmacists and the public in Vellore, South India. Indian Journal of Medical Ethics. 2013; 10(1), 20-27.
16. Gopalakrishnan S, Ganeshkumar P, Katta A. Assessment of prescribing practices among urban and rural general practitioners in Tamil Nadu. Indian Journal of Pharmacology. 2013; 45(3), 252-257.

17. Census of India. Ministry of home affairs: Government of India. 2011.

18. Centre for Good Governance. Poverty reduction at city level: Strategy development for Hyderabad. 2009.

19. Gopal Bhargava and Shankarlal Bhatt. Land and People of Indian states and union territories. Kalpaz Publication. 2006; P.132. ISBN 817835-358-X.

20. Kamla Gupta, Fred Arnold, and H.Lhungdim. Health and Living Conditions in Eight Indian Cities. National Family Health Survey (NFHS3), India, 2005-06. Mumbai: International Institute of Population Sciences; Calverton, Maryland, USA: ICF Macro. 2009.

21. Charmaz, K. Discovering' chronic illness: Using grounded theory. Social Science \& Medicine. 1990; 30(11), 1161-1172.

22. Kotwani A, Wattal Chand, Joshi, Pooran, Holloway K. Irrational use of antibiotics and role of the pharmacist: An insight from a qualitative study in New Delhi, India. Journal of clinical pharmacy and therapeutics.2011; 37. 308-12.

23. Kamat VR, Nichter M. Pharmacies selfmedication and pharmaceutical marketing in Bombay India. Social Science \& Medicine, 1998; 47:779-794.

24. Palmer NA, Dailey YM, Martin MV. Can audit improve antibiotic prescribing in general dental practice? Br Dent J. 2001; 191(5):253-5.

25. Chate RA, White S, Hale LR, Howat AP, Bottomley J, Barnet-Lamb J, Lindsay J, Davies TI, Heath JM. The impact of clinical audit on antibiotic prescribing in general dental practice. Br Dent J. 2006; 201(10): 635-41. 